\begin{tabular}{|c|c|c|}
\hline & Int.J.Curr.Microbiol.App.Sci (2016) 5(11): 741-750 & \\
\hline & International Journal of Current Microbiology and Applied Sciences & 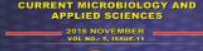 \\
\hline & ISSN: 2319-7706 Volume 5 Number 11 (2016) pp. 741-750 & \\
\hline $\begin{array}{l}\text { EXCELLENT } \\
\text { PUBLISHERS }\end{array}$ & & (1) \\
\hline
\end{tabular}

Original Research Article http://dx.doi.org/10.20546/ijcmas.2016.511.085

\title{
Neonatal Septicemia - Bacteriological Spectrum and Antibiogram-A Study from a Tertiary Care Center of North India
}

\author{
Varunika Vijayvergia*, Sunita Gupta and Jaswant Goyal \\ Department of Microbiology, JNU-IMSRC (Jaipur National University - Institute of Medical \\ Sciences and Research Center), India \\ *Corresponding author
}

\section{Keywords \\ Neonatal \\ septicaemia, \\ Antibiogram, neonatal intensive care unit (NICU).}

\begin{tabular}{l}
\hline Article Info \\
\hline Accepted: \\
26 October 2016 \\
Available Online: \\
10 November 2016
\end{tabular}

\section{A B S T R A C T}

Neonatal septicaemia, which is one of the leading cause of neonatal mortality and morbidity in India is characterised by systemic signs and symptoms of infection and accompanied with bacteraemia in first 4 weeks of life. To determine the bacterial spectrum, antimicrobial susceptibility pattern, and the level of contamination associated with the blood culture of the infants with neonatal septicemia in a tertiary level health care setup of North India. In this reterospective-prospective study, 65 blood samples were collected and processed for identification of the causative organism, from the clinically suspected cases of septicemia in neonatal intensive care unit (NICU). Antimicrobial susceptibility pattern of the isolates was obtained by Kirby Bauer disc diffusion method according to the Clinical and Laboratory Standard Institution (CLSI) recommendations. Actual rate of blood culture was observed and the number and nature of contaminants obtained from the blood cultures was also studied. Blood culture reports were positive in $32(49.23 \%)$ cases. Gram-negative septicaemia $(59.25 \%)$ was encountered more than Gram-positive (40.74\%). E.coli $(19.23 \%)$ was the predominant isolate followed by, Enterobacter spp and Coagulase negative staphylococcus (CoNS) in $15.38 \%$ cases each. Best overall sensitivity among Gramnegative isolates was to Colistin (94.44\%), Imipenem and Meropenam (83.33\%). Gram-positive isolates still showed excellent sensitivity to linezolid and vancomycin . Total rate of blood culture contamination came out to be $7.69 \%$ with CoNS being the most common contaminant. A rare pathogen Chrysobacterium indolegens was also isolated from one of the samples but later on it was found to be a contaminant in blood culture. Gram-negative organisms are the leading cause of neonatal septicaemia with E.coli being commonest. Coagulase negative Staphylococci is the predominant isolate among Gram-positive organisms but careful differentiation between the true pathogens and a mere culture contaminant is required since it is the most common contaminant as well. Most of the isolates are becoming resistant to common antibiotics therefore indiscriminate use of the higher end antibiotics like carbapenems and glycopeptides should be restricted.

\section{Introduction}

Neonatal sepsis is one of the most frequent causes of morbidity and mortality in newborns and is characterized by invasive bacterial infection that occurs in newborns between the first and the ninetieth day of the life (Schuchat et al., 2000). It is worldwide, 
incidence of sepsis in newborns varies between 1- 10 cases per 1000 live births, and the mortality varies between $15-50 \%$ (Vasiljević et al., 2008). Neonatal septicaemia is a clinical entity which is characterised by systemic signs and symptoms of infection and is accompanied by bacteraemia in first 4 weeks of life. The presentation of neonatal sepsis can be variable for different infants and can present with hypothermia, fever, lethargy, poor cry, refusal to suck or poor feeding, off colour, poor perfusion, shock, absent neonatal reflexes, bradycardia/ tachycardia, respiratory distress, hypo/ hyperglycaemia and metabolic acidosis. The etiology of septicemia is multi factorial. Neonatal sepsis is caused by a variety of Gram-positive as well as Gram negative bacteria and sometimes yeast (Gomaa et al., 2000). Uncontrolled use of various potent and broad spectrum antibiotics has led to emergence of resistant strains. This has become a major problem in various intensive care units for the treatment of critically ill patients. Therefore correct and timely identification of the causative organism along with the appropriate sensitivity pattern helps the clinicians to provide safe and effective therapies, develop rational prescription programmes which in due course of time will curtail the emerging antibiotic resistance overall. Inspite of the great advancement in the molecular diagnosis of bacterial and fungal infection, blood cultures still remain the mainstay of investigation for a potential case of sepsis in an infant. It is the Gold standard for isolation and verification of microbial pathogens in sepsis and also provide the antibiotic sensitivity profile of the organism which is the key to the correct and timely treatment of the patient. But for the judicious use of the procedure, ruling out the contamination is very important. Positive blood cultures, in a setting where such findings do not fit in the general condition of the child and where his/hers laboratory tests (i.e; C-reactive protein) are negative, is a common finding. False-positive blood cultures are usually a consequence of contamination of the blood samples by the bacteria from the skin and the environment of the child. False-positive blood cultures are a major problem to deal with, because such findings often lead to a falsely high morbidity which in turn adds to the patient and the cost of treatment. Premature infants with false-positive blood cultures usually are treated as children with sepsis.

The present study was conducted to determine the true spectrum of organisms associated with bacteraemia in neonates and to find out susceptibility pattern of pathogens causing neonatal sepsis so as to provide antibiogram to the clinicians for better patient management. Along with this an effort is also put to know the actual contamination rate of the blood cultures in this hospital and to define the common contaminants grown in blood cultures.

\section{Materials and Methods}

The present retrospective prospective study was conducted on all the 65 blood samples which came for culture from the neonates admitted in the neonatal intensive care unit of JNUIMSRC, which is a tertiary care hospital of north India, between the period of January 2016 to august 2016.

Approval for the study from the institutional ethical committee was obtained. Considering the fact that all the recommended sample collection and processing protocols are always being followed in the hospital, the data for the first (retrospective) part of the study was analyzed from the previous medical records of all the newborns treated in the NICU of 
JNUIMSRC from January to June 2016. In the prospective part of the study, the blood cultures of the neonates were actively followed up and correlated with the clinical condition of the neonate along with the other relevant lab investigations.

\section{Inclusion criteria}

All the blood samples which came for culture from the neonatal ICU were taken into study group except for ones which come under the exclusion criteria.

\section{Exclusion criteria}

Neonate with age more than 28 days or neonate on antibiotics prior to collection of blood were excluded from the study.

In all patients who had positive blood culture findings, cases were further looked for: the type of bacteria, and the site of the sample collection (peripheral vein, umbilical venous catheter). Blood cultures were considered as true positives when found in association with positive clinical findings such as tachypnoea, respiratory distress, apnoea, tachycardia, hypotension, hyper- or hypoglycaemia, metabolic acidosis, hypo- or hyperthermia, etc. and positive laboratory findings such as elevated levels of serum $\mathrm{C}$ reactive protein and/or procalcitonin, leucocytosis/ leucopoenia, thrombocytopenia, etc. Absence of all of these features even in the absence of any antibiotic administration, positive were suggestive of contamination in blood culture instead of true positive infection. In such cases a repeated blood culture was taken to determine the causative association and the isolate was considered pathogenic only if found repeatedly associated with the culture results.

Procedure of blood collection: About 1-2 ml of venous blood was drawn by a percutaneous venous puncture following strict aseptic precautions ( 3 swab technique) and aseptically inoculated into blood culture bottles containing 5-10 $\mathrm{ml}$ of brain heart infusion broth. Cultures were kept at $37^{\circ} \mathrm{C}$ for overnight incubation. Primary subcultures were done after $24 \mathrm{hrs}$ of incubation on to Blood agar \& Mac-Conkey agar plates. Even if the culture was sterile after overnight incubation, bottles were incubated further \& followed up by daily examination of the broth and doing a final subculture at the end of 7days (Puopolo et al., 2012) or at appearance of signs of growth, whichever was earlier. The positive growth was followed up by the conventional methods according to the standard operative procedures followed at our laboratory, including colony morphology, Gram's staining \& biochemical reactions. In case of any discrepancy in identification with conventional method, the help of some private based laboratory was taken for the automated identification. After the identification of the isolate, antimicrobial susceptibility testing was done by the conventional Kirby-Bauer disc diffusion method on Muller Hinton agar as per Clinical and Laboratory Standards Institute (CLSI) guidelines. The antibiotics which were used in our study were based on the hospital antibiotic policies (as per CLSI).

For descriptive statistics - absolute numbers, percentages and measures of central tendency (mean, median, standard deviation, minimum and maximum values) were used. For statistical testing, $\chi^{2}$ test and t-test were used as appropriate. In the applied tests, the level of significance were set at $95 \%$ $(\mathrm{p}<0.05)$.

\section{Results and Discussion}

During the study period, a total of 65 neonates with clinical sepsis were studied. 
Blood culture positivity was found in 32 cases $(49.23 \%)$ while 33 cases $(50.76 \%)$ were blood culture negative. But after clinical correlation 5 of the blood culture isolates were found to be contaminants as they were not compatible with the clinicopathological status of the patient. So finally the true blood culture positivity comes out to be of 27 isolates ie; $41.53 \% .26$ out of 27 were found to be the bacterial species and only one isolate was found to be the Candida species. None of the cases of neonatal septicaemia were found to be of poly-microbial aetiology. Out of the true culture positive cases, there were 16 $(59.25 \%)$ male and $11(40.74 \%)$ female neonates with the male-to-female ratio of 1.45. According to the time of onset of the sepsis, it was found that the cases of Earlyonset sepsis (EOS) cases higher (66.7\%) than the cases of the late onset sepsis (LOS).

Of the 26 bacterial isolates which were isolated, distribution of the isolates was as follows: E. coli 5, Enterobacter species 4, Coagulase negative staphylococcus (CoNS) 4, Klebsiella species 3, Pseudomonas species 3, Staphylococcus aureus 3, Citrobacter species 2, Burkholdaria species 1 and one Enterococcus species. So in total there were $18(69.23 \%)$ gram negative isolates and $8(30.76 \%)$ gram positive isolates from the blood culture. (figure.1)

Gram-positive organisms showed highest sensitivity to linezolid, and vancomycin, approaching upto $100 \%$ and least sensitivity to erythromycin (25\%) (figure.2). Gramnegative organisms showed good sensitivity to Colistin (94.44\%), Imipenem, Meropenam (83.33\%) and piperacillintazobactam (77.7\%). Cephalosporins showed poor sensitivity (figure.3).

Of the total 5 contaminants which were found in the blood culture most common was CONS ie; 3 , one was micrococci and 1 isolate was Chrysobacter indologenes which was identified with the help of VITEK 2(BD). None of these isolates were correlating well with the patient. It was identified that four out of these five cultures were took from an existing IV line on the baby. For further confirmation, repeat cultures were obtained from these patients and none of them came out to be positive. So these isolates were confirmed to be contaminants.

Neonatal septicemia is one of the most important causes of neonatal morbidity and mortality. The spectrum of causative agents in neonatal sepsis vary from place to place and the frequency of the causative organisms is variable in different hospitals and even in the same hospital at different times. For the effective management of neonatal septicemia cases, study of the bacteriological profile with their antibiotic susceptibility pattern plays a significant role.

Out of the total 65 clinically suspected cases of sepsis from NICU in this study, 27 blood cultures were true positive cases with a blood culture positivity rate of $41.53 \%$. According to the previous studies, blood culture positivity in neonatal septicaemia varies from $18.8 \%$ to $64.87 \%$. Studies conducted by Shah et al., (31.57\%), Dias and Vigneshwaran $(32 \%)$ and Mahapatra et al., (40\%) also showed the similar results as ours. On the other hand few studies conducted by Jyothi et al., (19.20\%), Rathod et al., (17.09\%) and Bhat et al., (17.80\%) showed much lower blood culture positivity rate. Low positivity might be due to the prior administration of the antibiotic before blood collection, possibility of infection with anaerobes, presence of fastidious organisms or lesser amount of the blood volume inoculated in the culture bottle. It was found that if the density of the 
organisms present is $<4$ colony forming units $(\mathrm{cfu}) / \mathrm{ml}$, blood volumes of $0.5 \mathrm{ml}$ or less had a significantly diminished chance of detecting bacteraemia. In the present study male babies (59.25\%) were affected more than female babies (40.74\%) with male to female ratio of 1.45 . It was similar to the findings of Kavita Nimboor et al., Sharma et al., and Garg et al., Male preponderance may be linked to the $\mathrm{X}$ linked immuneregulatory gene resulting in susceptibility to infections. It was found that in our study the incidence of EOS $(66.66 \%)$ was more than LOS $(33.33 \%)$. Our findings were similar with the reports from few previous studies. Higher incidence of EOS was also reported by Sheth et al., (56.52\%) and Nayak et al., $(70.66 \%)$. This could be due to immature immunologic response of neonates in the first week of life, making them more susceptible to infection in this period.

Gram-negative septicaemia (69.23\%) was encountered more than Gram-positive $(30.76 \%)$ in the present study which is comparable to studies conducted by Tsering et al., Shah et al., and Nayak et al., with 61, 67.17 and $61.33 \%$ cases of Gram-negative septicaemia, respectively. Among Gramnegative bacteria, we found E.coli as commonest isolate followed by Enterobacter species. It was contrary to some other studies by Chug et al., and Sharma et al., who reported Klebsiella spp as a predominant Gram-negative bacteria. In this study CoNS was the commonest isolate amongst Gram-positive cocci. This is in accordance with the study by Dias and Vigneshwaran et al., and Ballot et al., where also CoNS was found to be the commonest isolate among Gram positive organisms. CoNS is normally considered as a skin contaminant when isolated from blood. But the presence of CoNS in blood of the critically ill symptomatic babies, should never be ignored as contaminants as the clinical manifestation of CoNS sepsis can be varied and so they can be quite significant in such settings. In this study, we considered few CoNS as a true pathogen because the antibiotic sensitivity pattern of these CoNS also suggested so and these also correlated well clinically.

In this study antibiotic susceptibility pattern revealed that majority of Gram-negative bacteria were sensitive to Colistin (94.44\%), Imipenem (83.33\%) and Meropenam $(83.33 \%)$ which is comparable to the study done by Jyothi et al., Among aminoglycosides maximum sensitivity was seen to Amikacin (72.22\%) which is similar with Madavi et al., Least sensitivities were found with cefuroxime and ciprofloxacin in this study. The Grampositive bacteria showed good sensitivity to linzolid and vancomycin approaching upto $100 \%$ which is comparable to study by Jagoo et al., Shah et al., and Sheth et al., All the isolates of Staphylococcus were sensitive to linezolid and vancomycin. Vancomycin sensitive Enterococcus spp were reported in our NICUs. Finding of VRE could be a problem since these bacteria are resistant to all available antibiotics and have the potential of being reservoirs for glycopeptide-resistant genes that can be transferred to other more virulent pathogens. Multi drug resistance to antimicrobial agents was found in the present study. Cefoxitin resistance was seen in $62.5 \%$ of Staphylococcal isolates suggesting the presence of about $37.5 \%$ of MRSA in this hospital . Our results were compatible to few other studies. Sheth et al., and Shah et al., reported MRSA in 26.67 and 50\% isolates, respectively.

In this study 5 samples with growth obtained were considered as contaminants on clinicopathological correlation with patient's findings. So the total rate of contamination of the blood culture in our hospital was 
found to be $7.69 \%$. Literature suggests that the actual rates of blood culture contamination seem to vary widely between institutions, from as little as $0.6 \%$ to over 6\% (Jajoo et al., 2015; Lee et al., 1967). It has been found to be slightly higher $(7.69 \%)$ in our setting. But the evidence suggests that contamination occurs more frequently in the peadiatric population, particularly in young infants (Norberg et al., 2003; Weinbaum et al., 1997). Higher rates of contamination can also be attributed to the manual method of blood culture followed at this institution. In an effort to reduce unnecessary discomfort, pediatricians often use existing intravenous catheters for obtaining cultures instead of peripheral venipuncture. This may also lead to higher chances of contamination. Trained phlebotomy or blood culture teams have been found to decrease blood culture contamination rates.
Amongst the 5 contaminants obtained in the blood cultures one was Chryseobacterium indologenes which is a rare human pathogen but finding it as a contaminant in this study highlights the fact that all rare organisms are not pathogenic and clinical correlation needs to be done to avoid injudicious use of antibiotics due to contamination with these multidrug resistant contaminating bacteria. Chryseobacterium spp. can survive in chlorinated waters, and in the hospital environment they exist in water systems and wet surfaces and serve as potential reservoirs of infection. Colonization of patients via contaminated medical devices such as respirators, endotracheal and tracheostomy tubes, humidifiers, incubators for newborns and syringes has been documented previously.

Fig.1

\section{Distribution of the isolates in blood cultures}

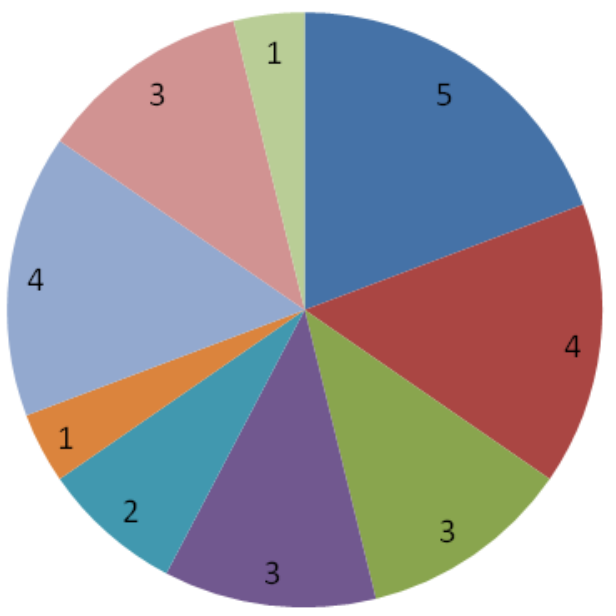

E.coli

Enterobacter

Klebsiella

Pseudomonas

Citrobacter

- Burkholdaria

CONS

Staph aureus

Enterococcus 
Fig.2

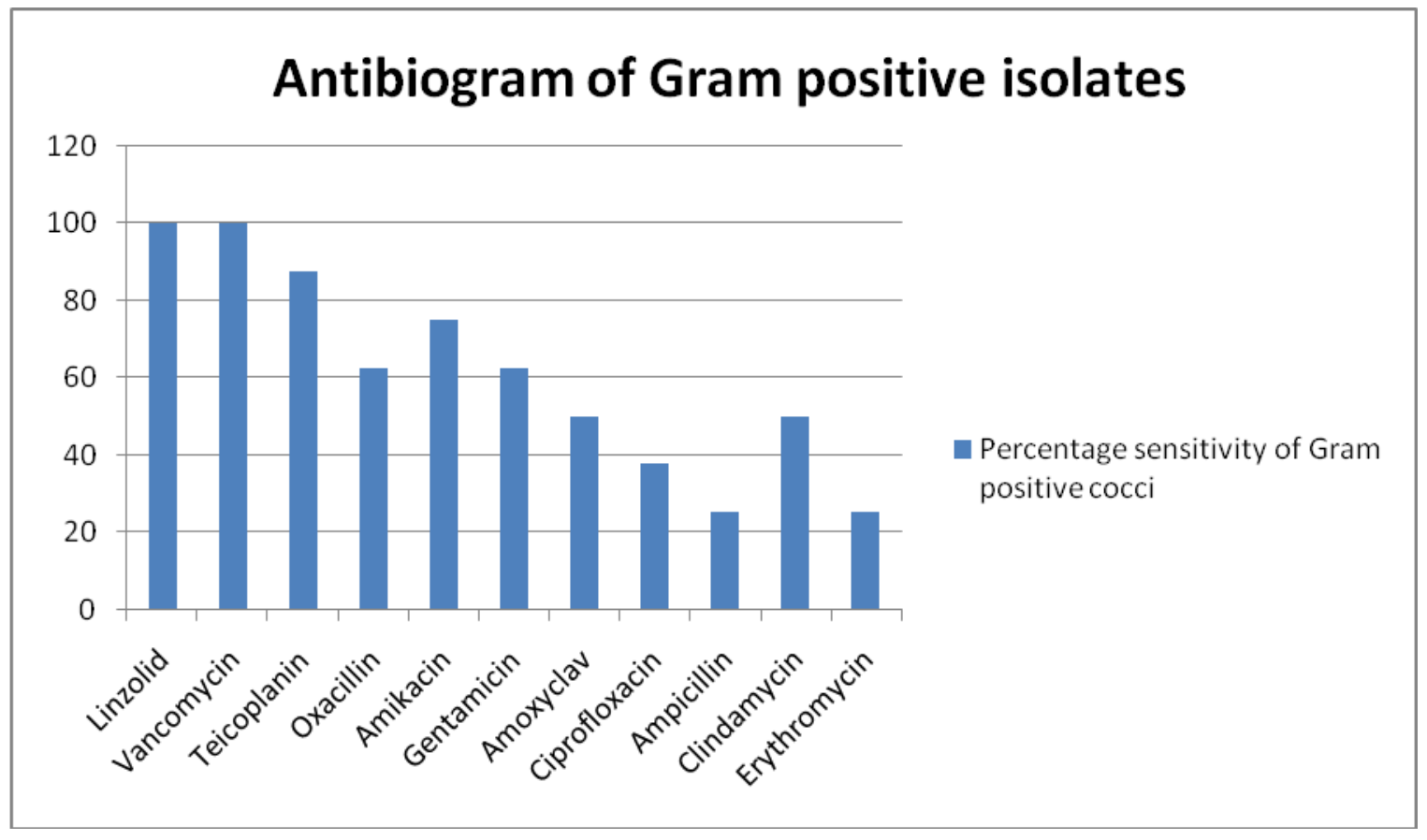

Fig.3

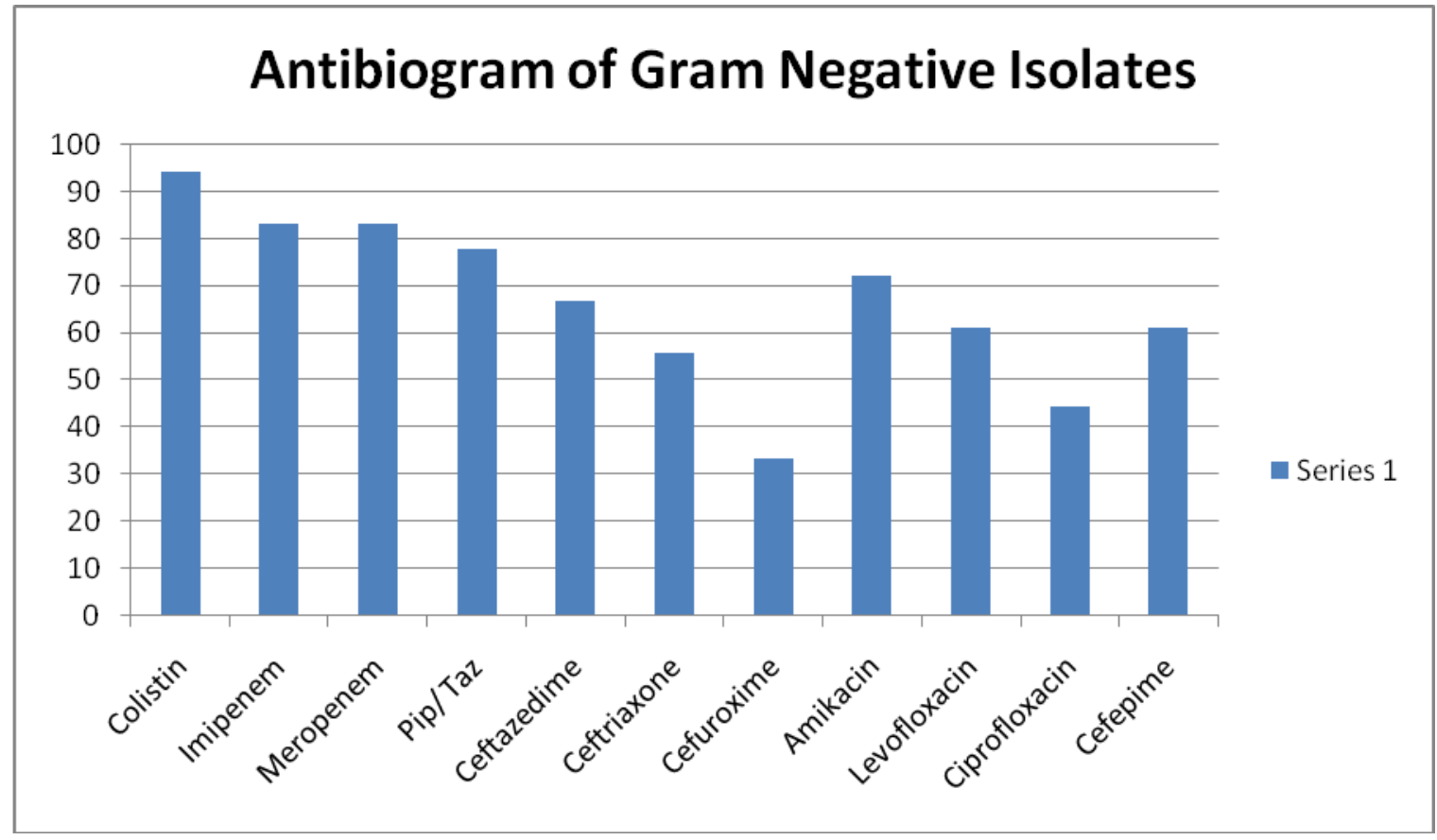

Chryseobacterium infections in humans are usually acquired nosocomially and are frequently associated with the presence of invasive equipments (intra-vascular catheters, endotracheal tubes, prosthetic device) in immunocompromised patients or patients who have received long-term broadspectrum antibiotics (Nulens et al., 2001; 
Lin et al., 2003). So, it becomes important to identify the actual contamination rate of blood cultures at every hospital level and also to identify the true nature of the organisms. To distinguish sepsis from culture contamination in young infants, quantitative blood cultures can be performed in conjunction with specific clinical information to identify the true pathogens. But it was not done in our study. Moreover taking a larger number of samples in the study would give us a better understanding of the true sensitivity pattern and the spectrum of infecting microorganisms.

In conclusion, effective use of blood cultures in paediatric practice is a key component for the management of sepsis. But the higher susceptibility of the newborns to the infection, owing to the immaturity of their immune system, makes automatic extrapolation of the adult data to the newborns difficult and potentially unfounded. So the continuous monitoring of the positivity in the blood cultures in this neonatal population is required for timely administration of effective empirical therapy based on antibiogram and the spectrum of the infecting microbes at every center. A great progress has been made in improving the ability to distinguish contamination from true bacteremia. But better strategies for preventing contamination in the first place, are required to be implemented very strictly. Along with this, further research on the value of time to positivity and quantity of growth for differentiating culture contamination from bacteremia is necessary.

\section{References}

Ballot, D.E, Nana T, Sriruttan C, Cooper PA. Bacterial bloodstream infections in neonates in a developing country. ISRN Pediatr, 508512.
Bhat, Y.R., Lewis, L.E., K.E.V. 2004. Bacterial isolates of early-onset neonatal sepsis and their antibiotic susceptibility pattern between 1998 and 2004: an audit from a center in India. Ital. J. Pediatr., 37: 32.

Chugh, K., Aggarwal, B.B., Kaul, V.K., Arya, S.C. Bacteriological profile of neonatal septicemia. Indian J Pediatr 1988;55:961-5.

Clinical and Laboratory Standards Institute. Performance standards for antimicrobial susceptibility testing; 23rd informational supplement. Pennsylvania: Clinical and Laboratory Standards Institute, p.M100-S23.

Dias, E., Vigneshwaran, P. 2010. The bacterial profile of neonatal septicaemia in a rural hospital in South India. J. Clin. Diagn. Res., [Serial Online] 4:3327-30. Available from http://www.jcdr.net/back_issues.asp?is $\operatorname{sn}^{1} / 4097309 x$

\&year $1 / 42010 \&$ month $1 / 4$ December\&vol ume $1 / 44 \&$ issue $1 / 46 \&$ page- $\quad 1 / 43327$ 3330\&id $1 / 41008$.

Du Moulin, G.C. 1979. Airway colonization by Flavobacterium in an intensive care unit. J Clin Microbiol., 10: 155-160.

Garg, D., Agarwal, N. 2014. Aetiology and presentation of neonatal septicaemia at Tertiary Care Hospital of Southern Rajasthan. Int. J. Med. Sci. Edu., 1: 12-20.

Gomaa, H.H.A., Udo, E.E., Rajaram, U. 2001. Neonatal septicaemia in AlJahra hospital Kuwait: etiologic agents and antibioticsensitivity patterns. Med. Princ. Pract., 10: 145-50.

Jajoo, M., Kapoor K, Garg LK, et al. To study the incidence and risk factors of early onset neonatal sepsis in an out born neonatal intensive care unit of India. J Clin Neonatol 2015;4:91-5.

Jyothi, P., Basavaraj, M.C., Basavaraj, P.V. 2013. Bacteriological profile of 
neonatal septicemia and antibiotic susceptibility pattern of the isolates. $J$. Nat. Sci. Biol. Med., 4: 306-9.

Lee, S., I. Schoen, and A. Malkin. 1967. Comparison of use of alcohol with that of iodine for skin antisepsis in obtaining blood cultures. Am. J. Clin. Pathol. 47:646-648.

Lin JG, Wang WS, Yen CC, Liu JH, Chiou TJ, Yang MH, Chao TC, Chen PM: Chryseobacterium indologenes bacteremia in a bone marrow transplant recipient with chronic graftversus-host disease. Scand J. Infect. Dis., 35: 882-883.

Little, J. R., P. R. Murray, P. S. Traynor, and E. Spitznagel. 1999. A randomized trial of povidone-iodine compared with iodine tincture for venipuncture site disinfection: effects on rates of blood culture contamination. Am. J. Med. 107:119-125.

Madavi, D., Aziz F, Agrawal G. Clinicobacteriological profile and antibiotic sensitivity pattern of neonatal septicaemia- a prospective observational study. Int J Cur Res Rev 2015;7:13-20.

Mahapatra, A., Ghosh, S.K., Mishra, S., et al. 2002. Enterobacter cloacae: a predominant pathogen in neonatal septicaemia. Indian J. Med. Microbiol., 20: 110-12.

Mandell GL, Dolin R: Principles and Practice of Infective Disease. 6 edition. New York: Elsevier; 2005, 2757-2759.

McGowan, J. E., Jr., L. Bratton, J. O. Klein, and M. Finland. 1973. Bacteremia in febrile children seen in a "walk-in" pediatric clinic. N. Engl. J. Med. 288:1309-1312.

Nayak, S., Rai, R., Kumar, V.K., et al. 2014. Distribution of microorganisms in neonatal sepsis and antimicrobial susceptibility patterns in a tertiary care hospital. Arch. Med. Health Sci., 2: 136-9.

Nimboor, K., Ravindranath. 2006. Bacteriological (aerobic) study of neonatal septicemia in NICU. Dissertation Doctor of Medicine.

Norberg, A., N. C. Christopher, M. L. Ramundo, J. R. Bower, and S. A. Berman. 2003. Contamination rates of blood cultures obtained by dedicated phlebotomy vs intravenous catheter. JAMA 289:726-729.

Nulens E, Bussels B, Bols A, Gordts B, Van Landuyt HW: Recurrent bacteremia by Chryseobacterium indologenes in an oncology patient with a totally implanted intravenous device. Clin Microbiol Infect 2001, 7: 391-393.

Ohlin, A. 2011. What is neonatal sepsis? Acta Pediatr., 100: 7-8.

Puopolo, K.M. 2012. Bacterial and fungal Infection. In: Cloherty JP, Eichenwald EC, Hansen AR, Stark AR. Manual of Neonatal care. 7th edn. Philadelphia: Wolter Kluwer Lippincott, 624-55.

Rathod, S.D., Bhatia, P.V., Patel, P.H., et al. 2012. Bacteriological analysis and resistance pattern among various culture isolates from neonatalsepticemia at Tertiary Care Hospital, Ahmedabad. Natl. J. Med. Res., 466-9.

Schelonka, R.L., Chai, M.K., Yoder, B.A., et al. 1996. Volume of blood required to detect common neonatal pathogens. J. Pediatr., 129: 275-8.

Schifman, R. B., and A. Pindur. 1993. The effect of skin disinfection materials on reducing blood culture contamination. Am. J. Clin. Pathol. 99: 536-538.

Schifman, R. B., C. L. Strand, F. A. Meier, and P. J. Howanitz. 1998. Blood culture contamination: a College of American Pathologists Q-Probes study involving 640 institutions and 497134 
specimens from adult patients. Arch. Pathol. Lab. Med. 122:216-221.

Schuchat, A., Zywicki, S.S., Dinsmoor, M.J. 2000. Risk factors and opportunites for prevention of early-onset neonatal sepsis, a multicenter case-control study. Pediatrics, 105(1), 21-26.

Shah, A., Mulla, S., Revdiwala, S. 2012. Neonatal sepsis: high antibiotic resistance of the bacterial pathogens in a neonatal intensive care unit of a tertiary Care hospital. J. Clin. Neonatol., 1: 72-5.

Shah, H.Y., Gadhavi, H.M., Shah, V.P., et al. Antibiotics Susceptibility Patterns of Bacterial Isolates Among Neonatal Septicemia in Tertiary Care Hospital, Jamnagar, Gujarat. Global Res. Analysis 1: 99-100.

Sharma, A., Kutty, C.V., Sabharwal, U., et al. 1993. Diagnostic and prognostic role of CRP and $\mathrm{m}$-ESR in neonatal septicemia. Indian Pediatr., 30: $347-$ 50.

Sheth, K.V., Patel, T.K., Tripathi, C.B. 2012. Antibiotic sensitivity pattern in neonatal intensive care unit of a tertiary care hospital of India. Asian J. Pharm. Clin. Res., 5: 46-50. DOI: 10.3109/14767058.2016.1152251

Microbial-antimicrobial pattern of neonatal sepsis.
Shrestha, S., Adhikari, N., Rai, B.K., Shreepaili, A. 2010. Antibiotic resistance pattern of bacterial isolates in neonatal care unit. JNMA J. Nepal Med. Assoc., 50: 277-81.

Tallur, S.S., Kasturi, A.V., Nadigr, S.D., Krishna, B.V. 2000. Clinicobacteriological study of neonatal septicemia in Hubli. Indian J. Pediatr., 67: 169-74.

Tsering, D.C., Chanchal, L., Pal, R., Kar, S. 2011. Bacteriological profile of septicemia and the risk factors in neonates and infants in Sikkim. $J$. Glob. Infect. Dis. Infect., 3: 42-5.

Vasiljević, B., Antonović, O., MaglajlićĐukić, S., Gojnić, M., Vrednosti, Creaktivnog proteina kod novorođenčadi sa sepsom. Srpski arhiv za celokupno lekarstvo. 2008; 136(56), 253-257.

Weinbaum, F. I., S. Lavie, M. Danek, D. Sixsmith, G. F. Heinrich, and S. S. Mills. 1997. Doing it right the first time: quality improvement and the contaminant blood culture. J. Clin. Microbiol. 35: 563-565.

Weinstein, M.P. 2003. Blood culture contamination: persisting problems and partial progress. J. Clin. Microbiol., 41: 2275-2278.

\section{How to cite this article:}

Varunika Vijayvergia, Sunita Gupta and Jaswant Goyal. 2016. Neonatal Septicemia Bacteriological Spectrum and Antibiogram-A Study from a Tertiary Care Center of North India. Int.J.Curr.Microbiol.App.Sci. 5(11): 741-750. doi: http://dx.doi.org/10.20546/ijcmas.2016.511.085 